\title{
Erythropoietin facilitates the recruitment of bone marrow mesenchymal stem cells to sites of spinal cord injury
}

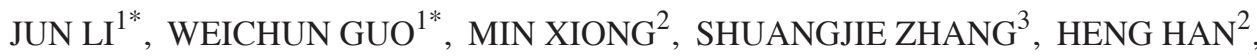 \\ JIE $\mathrm{CHEN}^{2}$, DAN MAO ${ }^{2}$, HUALONG YU ${ }^{2}$ and YUN ZENG ${ }^{2}$ \\ ${ }^{1}$ Department of Orthopedics, Renmin Hospital of Wuhan University, Wuhan, Hubei 430060; \\ ${ }^{2}$ Department of Orthopedics, Dongfeng General Hospital of Hubei University of Medicine, Shiyan, Hubei 442008; \\ ${ }^{3}$ Department of Urology, Taihe Hospital of Hubei University of Medicine, Shiyan, Hubei 442000, P.R. China
}

Received June 12,2015; Accepted July 26, 2016

DOI: $10.3892 / \mathrm{etm} .2017 .4182$

\begin{abstract}
Despite the successes of bone marrow mesenchymal stem cell (BMSC) transplantation for the treatment of spinal cord injuries, only a small fraction of grafted cells migrate to the target areas. Therefore, there remains a need for more efficient strategies of BMSC delivery. The present study was designed to explore this. Rat models of spinal cord injury (SCI) were established and exposed to phosphate buffered saline (control), BMSCs or BMSCs + erythropoietin (EPO). Basso, Beattie and Bresnahan (BBB) locomotor scale and grid walk tests were then utilized to estimate neurological rehabilitation. Additionally, the following assays were performed: Immunofluorescence localization of BMSCs to the site of SCI; the transwell migration assay to detect in vitro cellular migration; the terminal deoxynucleotidyl transferase dUTP nick end labeling assay to determine the apoptotic index of the lesion; and western blotting analysis to evaluate the expression of vascular endothelial growth factor (VEGF) and brain derived neurotrophic factor (BDNF) at the site of $\mathrm{SCI}$. The BBB scores of the BMSC + EPO treated group were significantly increased compared with the BMSC treatment group $(\mathrm{P}<0.05)$. For example, BMSC + EPO treated rats had a significantly decreased number of hind limb slips compared with the BMSC treatment group $(\mathrm{P}<0.05)$. Furthermore, EPO significantly increased the migration capacity of BMSCs compared with the control group $(\mathrm{P}<0.001)$. In addition, the apoptotic index of the BMSC + EPO group was significantly decreased compared with the BMSC group $(\mathrm{P}<0.05)$. Green fluorescent protein-labeled BMSCs were detected at the site of
\end{abstract}

Correspondence to: Dr Min Xiong, Department of Orthopedics, Dongfeng General Hospital of Hubei University of Medicine, 16 Daling Road, Shiyan, Hubei 442008, P.R. China

E-mail: mrorthopedics@sina.com

*Contributed equally

Key words: erythropoietin, bone marrow, mesenchymal stem cells, spinal cord injury
SCI in the BMSC and BMSCs + EPO groups, with the signal being notably stronger in the latter. Moreover, the expression of VEGF and BDNF in the BMSCs + EPO group was significantly increased compared with the BMSC group $(\mathrm{P}<0.05)$. In conclusion, the results of the present study indicate that EPO can facilitate the recruitment of BMSCs to sites of SCI, increase expression of BDNF and VEGF, and accelerate recovery of neurological function following SCI.

\section{Introduction}

Spinal cord injury (SCI), which can result from traumatic injuries and progressive neuropathies, is a devastating condition that causes physical and emotional damage to individuals, and places an economic burden on society (1). SCI triggers a cascade of secondary damage, including ischemia, hypoxia, necrocytosis, inflammation and exacerbation of axon demyelination, which causes substantial neurological disability in the affected areas of the extremities and/or trunk, such as loss of bowel, bladder and sexual functions (2,3). Despite advances in medical and surgical technique and regenerative engineering, no current treatments can reverse the devastating consequences of SCI (4).

Previous studies in rodent models identified that transplantation of stem cells could ameliorate secondary damage caused by SCI and facilitate functional recovery (5). The capacity of stem cells to initiatively home into regional tissue or passively home into lesion area supports the principle for the targeted delivery (6). Among candidate stem cells, BMSCs have emerged as a promising alternative to adipose-derived stem cells induced pluripotent stem cells and embryonic stem cells, demonstrating good axonal sprouting and regrowth in a SCI (7). Accumulating evidence indicates that BMSCs can promote the repair and regeneration of neurons, and promote functional rehabilitation, in animal models of SCI (8). A number of characteristics of BMSCs make them suitable for transplantation, including being easy to isolate and obtain, their multi-lineage potential, immunosuppression and suitability for autologous transplantation meaning there are no ethical issues (9). Despite the advances in BMSC transplantation in the last decades, only a minority of grafted cells migrate to target areas and BMSC survival is decreased due to the route 
of administration (venous system transplantation) as, after being filtered by circulatory system and blood brain barrier, only a small portion of transplanted BMSCs are able to reach the injured spinal cord (10). A previous study determined that free radical generation, deficiency in trophic factors and cell apoptosis result in poor survival of transplanted BMSCs (11). Therefore, more efficient strategies for BMSC delivery need to be explored in order to obtain the best possible therapeutic outcome.

Erythropoietin (EPO), which is known for its tissue protective and regenerative capabilities for a number of organs, such as the heart, spinal cord, brain and kidneys, has been shown to facilitate BMSC recruitment and angiogenesis (12-15). Interestingly, the results of a recent study demonstrate that the EPO receptor is expressed on the surface of BMSCs and via this EPO could promote the proliferation of BMSCs in acute kidney injury and reverse their low secretion (BMSCs can be secreted autologously by rats with acute kidney injury, but this rare, and EPO may promote the secretion of BMSC) (16). In addition, a recent study found that EPO promote the mobilization of BMSCs to damaged bone tissue and trigger BMSC differentiation in osteogenesis (17). The current study hypothesized that BMSC targeting to sites of SCI could be enhanced by co-transplantation with EPO, facilitating enhanced functional recovery. To investigate this hypothesis and, if true, explore the possible mechanisms through which it occurs, a rat model of SCI was created through the improved Allen method (18).

\section{Materials and methods}

Experimental animals. Pathogen-free female Sprague Dawley rats ( $\mathrm{n}=60$; age, 8 weeks old; weight, 200-250 g) were provided by the Animal Experiment Center at Hubei University of Medicine (Shiyan, China). The rats were housed with 3 animals cage from $\sim 1$ week prior to the commencement of the experiments, with free access to food and water and were maintained in a suitable environment at $21^{\circ} \mathrm{C}, 60 \%$ air humidity and a $12 \mathrm{~h}$ light/dark cycle. The majority $(n=45)$ of the rats were used to establish models of SCI and the remaining rats $(n=15)$ were used to derive BMSCs for culture. All procedures involving animals were approved by Laboratory Animal Management Committee of Hubei University of Medicine (Shiyan, China).

Derivation and culture of BMSCs. BMSCs were derivatized according to a previously described method (19). Briefly, rats were sacrificed by decapitation following anesthesia $(0.8 \mathrm{ml}$ $10 \%$ chloral hydrate; SouthernBiotech, Birmingham, AL, USA). Then, femurs and tibiae were removed and conterminal tissues withdrawn in a sterile environment. Bone marrow tissues were rinsed with phosphate buffer saline (PBS) and the BMSCs were isolated. Retrieved BMSCs were centrifuged at $1,000 \times \mathrm{g}$ for $5 \mathrm{~min}$ at room temperature and cultured in Dulbecco's modified Eagle medium (DMEM; Invitrogen; Thermo Fisher Scientific, Inc., Waltham, MA, USA) supplemented with $10 \%$ fetal bovine serum (FBS; HyClone; GE Healthcare Life Sciences, Logan, UT, USA). Culture media was replaced every 3 days. BMSCs were sub-cultured when they were between 80 and $90 \%$ confluence. To track the in vivo migration of BMSCs towards the site of SCI, plasmid transfection of a recombinant adenovirus encoding green fluorescent protein (GFP) was performed according to the manufacturer's instruction (Ad-GFP; Cyagen, Sunnyvale, CA, USA). Culture media was replaced $6 \mathrm{~h}$ following transfection and expression of GFP was confirmed 2 days following the media being replaced via fluorescence microscopy.

Establishing the rat model of SCI. Rats were anesthetized with an intraperitoneal injection of $10 \%$ chloral hydrate $(0.3 \mathrm{ml} / 100 \mathrm{~g})$ and fixed in a prone position using a vertical locator (W.M. Keck Center for Collaborative Neuroscience, Rutgers University, New Brunswick, NJ, USA). The ninth thoracic vertebra crest was identified and $\sim 2 \mathrm{~cm}$ of the skin and superficial fascia over this region was incised along the posterior median line under aseptic conditions. A dorsal laminectomy was performed on the ninth thoracic vertebra crest and the corresponding spinal cord unfolded. Subsequently, a moderate compressional SCI was imposed on each rat by perpendicularly descending a spinal cord impactor (W.M. Keck Center for Collaborative Neuroscience Rutgers, New Brunswick, NJ, USA) with a $10 \mathrm{~g}$ impact rod from a height of $5 \mathrm{~cm}$ onto the spinal cord and compress it for $10 \mathrm{sec}$. The following signs indicated that this procedure was successful: Rat tails twitched involuntary, bilateral hind limbs and truncus convulsed, both hind limbs were flaccid and there was paralysis of the hind limbs. Following surgery, the incision was irrigated with penicillin and saline and the muscles and skin were stitched in their correct anatomical layers.

Groupings and treatment. SCI model rats $(\mathrm{n}=45)$ were randomly distributed into the following three groups: A, the control group, treated once with $10 \mu \mathrm{l}$ PBS by intraspinal injection; B, the BMSC group, treated with $10 \mu \mathrm{l}$ of BMSC suspension containing $3 \times 10^{5}$ cells by intraspinal injection; group $\mathrm{C}$, the BMSC + EPO group, treated with $10 \mu \mathrm{l}$ of BMSCs suspension containing $3 \times 10^{5}$ cells and recombinant human EPO (rhEPO; Beijing Four Rings Bio-Pharmaceutical Co., Ltd, Beijing, China) through intraperitoneal injection $\left(5 \times 10^{3} \mathrm{IU} / \mathrm{kg}\right)$. Rats were injected intramuscularly with sulfentanil $(0.05 \mu \mathrm{g} / \mathrm{kg})$ and penicillin $\left(3 \times 10^{4} \mathrm{U} / \mathrm{kg}\right)$ for the first 3 days after SCI. The bladders of the rats were pressed by hand twice daily until automatic micturition function was restored. Food and water were places in areas accessible to the rats.

Evaluation of neurological function. Hind limb motor function was quantified and recorded at 1, 7, 14, 21 and 28 days following SCI with reference to the Basso, Beattie and Bresnahan (BBB) locomotor scale (20). The BBB scale range is between 0 and 21 , where 0 stands for complete loss of locomotive function and 21 stands for normal locomotive function. A $120 \times 30 \mathrm{~cm}$ foam-padded box with a flat bottom was used for the BBB test and rats were placed in the box 2 days prior to evaluation to acclimatize to their surroundings.

Additionally, a grid walk test was performed to evaluate motor sensitivity and capacity for precise control of the hind limbs. Rats were allowed to crawl freely in a 120x120 cm grid structure, which was divided into $10 \mathrm{~mm}$ squares. Missteps were tracked as the rats mislaid the paw through the squares. The tests was terminated when a maximum of 20 missteps were achieved. Rats that failed to accomplish a coherent gait 
in the BBB scale test were not put through the grid walk test and were instead assigned the maximum score (21). The individuals recording the movements were blinded to the study to reduce error and raise accuracy.

Detection of in vitro BMSC migration. BMSC migration was detected using the transwell migration assay (cat no. 3413; Beijing Unique Biotechnology Co., Ltd, Beijing, China). In the chemotaxis group, serum-free medium $(200 \mu \mathrm{l})$ with $1 \times 10^{5}$ (cells $\left./ \mathrm{ml}\right)$ BMSCs was placed into the upper chambers, while DMEM $(800 \mu \mathrm{l})$ with 10 units rhEPO and 10\% FBS was put into the lower chambers. In the control group, the same procedure was followed, except that rhEPO was excluded from the media in the lower chamber. Following $18 \mathrm{~h}$ of incubation as previously described, cells that failed to migrate from the upper chamber to the lower chamber were washed away. Then, cells that had migrated on to the membranes were fixed with $4 \%$ paraformaldehyde for $30 \mathrm{~min}$, stained with $5 \%$ crystal violet for $20 \mathrm{~min}$, washed with PBS, and observed and imaged using an optical microscope. Cells were counted three times, and an average was taken.

Detection of apoptosis at the site of SCI. The apoptotic index of the SCI lesion site was determined using the terminal deoxynucleotidyl transferase dUTP nick end labeling (TUNEL) assay using an in situ cell death detection kit (cat no. 40306ES50; Po Valley Biotechnology, Shanghai, China) according to the manufacturer's instructions. The specimens from the spinal cord were frozen and embedded in optical coherence tomography (Leica Biosystems, Shanghai, China), and $7 \mu \mathrm{m}$ longitudinal cryostat sections were cut. Subsequently they were dewaxed, hydrated with a series of graded alcohol (99.5\%) and co-cultured with proteinase $\mathrm{K}$ at room temperature for $30 \mathrm{~min}$, followed by devitalization of endogenous peroxidases with $30 \% \mathrm{H}_{2} \mathrm{O}_{2}$ for $5 \mathrm{~min}$. Then, sections were incubated with the TUNEL reaction mixture in a $60 \%$ air humidity atmosphere at $37^{\circ} \mathrm{C}$ for $60 \mathrm{~min}$, followed by suspension of the reaction using the stop buffer. Subsequent staining with hematoxylin dyed the nuclei of apoptotic cells brown. The apoptotic index was calculated as the percentage of TUNEL-positive (apoptotic) cells out of the total number of nucleated cells. Cells were counted three times, and an average was taken.

Detection of in vivo BMSC distribution. To detect the distribution of BMSCs in the injured spinal cord of rats, fluorescence microscopy scanning was conducted on samples taken 4 weeks following surgery. Samples (as previously described) were fixed with acetone for 20-30 min and then embedded with a 1x1x1 cm foil paper mold, which was filled with frozen embedding agent. Subsequently, they were frozen at $-80^{\circ} \mathrm{C}$ in liquid nitrogen, and then cut into slices with freezing microtome (Thermo Fisher Scientific, Inc.). Then, sections were blocked with 5\% normal goat serum (Beyotime Institute of Biotechnology, Beijing, China) at room temperature for $30 \mathrm{~min}$. Sections were blotted using bibulous paper to remove excess liquid and mounted onto slides with an anti-fluorescent quencher (Antifade Mounting Medium; Leica Biosystems, Shanghai, China). The distribution of BMSCs was determined through visualization of GFP signals using fluorescence microscopy (Shanghai Wanheng Precision Instruments Co., Ltd., Shanghai, China).

Western blot analysis of the expression of vascular endothelial growth factor (VEGF) and brain derived neurotrophic factor (BDNF) in vivo. Samples (as previously described) were homogenized using Tissue Tearor (BioSpec Products, Inc., Bartlesville, OK, USA) for $1 \mathrm{~min}$ at $4^{\circ} \mathrm{C}$, following lysis in radioimmunoprecipitation buffer (Boster Biological Technology, Ltd., Wuhan, China). Then, samples underwent centrifugation $(12,000 \mathrm{xg})$ at $4^{\circ} \mathrm{C}$ for $40 \mathrm{~min}$ and $50 \mu \mathrm{g}$ of total protein was extracted from the supernatant for separation by $10 \%$ SDS-PAGE, followed by transfer to a PVDF membrane (HD Biosciences Co., Ltd., Shanghai, China). After being blocked with Tris Buffered Saline Tween-20 (TBST; EMD Millipore, Billerica, MA, USA) for $30 \mathrm{~min}$, the PVDF membranes were incubated at $4^{\circ} \mathrm{C}$ overnight with primary monoclonal antibodies as follows: Rabbit anti-VEGF (cat no. CL-1313T), rabbit anti-BDNF (cat no. AHP1831) or rabbit anti-GAPDH antibodies (cat no. A01020) (all diluted 1:200; HD Biosciences Co., Ltd., Shanghai, China). Subsequently, they were washed with TBST for $30 \mathrm{~min}$, incubated with horseradish peroxidase-labeled goat anti-rabbit antibody (1:5,000; cat no. DC06L-200UG; Shanghai Biological Technology Co., Ltd., Shanghai, China) at $26^{\circ} \mathrm{C}$ for $2 \mathrm{~h}$. Bands were then visualized using an enhanced chemiluminescence detection kit (cat no. 36222ES60; Thermo Fisher Scientific, Inc., Waltham, MA, USA) and X-ray film. The densitometry of the bands was analyzed using Image-Pro Plus 6.0 software (Media Cybernetics, USA). Cells were counted three times, and an average was taken.

Statistical analysis. All results are expressed as the mean \pm standard deviation. One-way analysis of the variance was used to compare the results of different groups. Multiple comparison between the groups was performed using Student Newman Keuls method. Statistical analysis was performed using SPSS software (version 21.0; IBM SPSS, Armonk, $\mathrm{NY}, \mathrm{USA}$ ). $\mathrm{P}<0.05$ was considered to indicate a significant difference.

\section{Results}

EPO enhances the recovery of neurological function in rat models of SCI treated with BMSCs. Functional hind limb movement was measured using two ethological examinations. The BBB locomotor rating scale was employed to access motor function in each group post-SCI (Fig. 1A). Due to the effect of the anesthetic used prior to surgery, no significant difference was found between the three groups 1 day following SCI (Fig. 1A). As time passed, the groups all showed increasing motor function, however, the BBB score of the BMSC + EPO group was significantly higher compared with that of the control $(\mathrm{P}<0.01)$ and BMSC alone groups $(\mathrm{P}<0.05)$ (Fig. 1A). In addition, the grid walk test was performed to access the coordination and accuracy of hind limb motor function (Fig. 1B). BMSC + EPO treated rats showed significantly less missteps from 7 days post-SCI compared with the control $(\mathrm{P}<0.01)$ and $\mathrm{BMSC}$ alone groups $(\mathrm{P}<0.05)$ (Fig. 1B). 

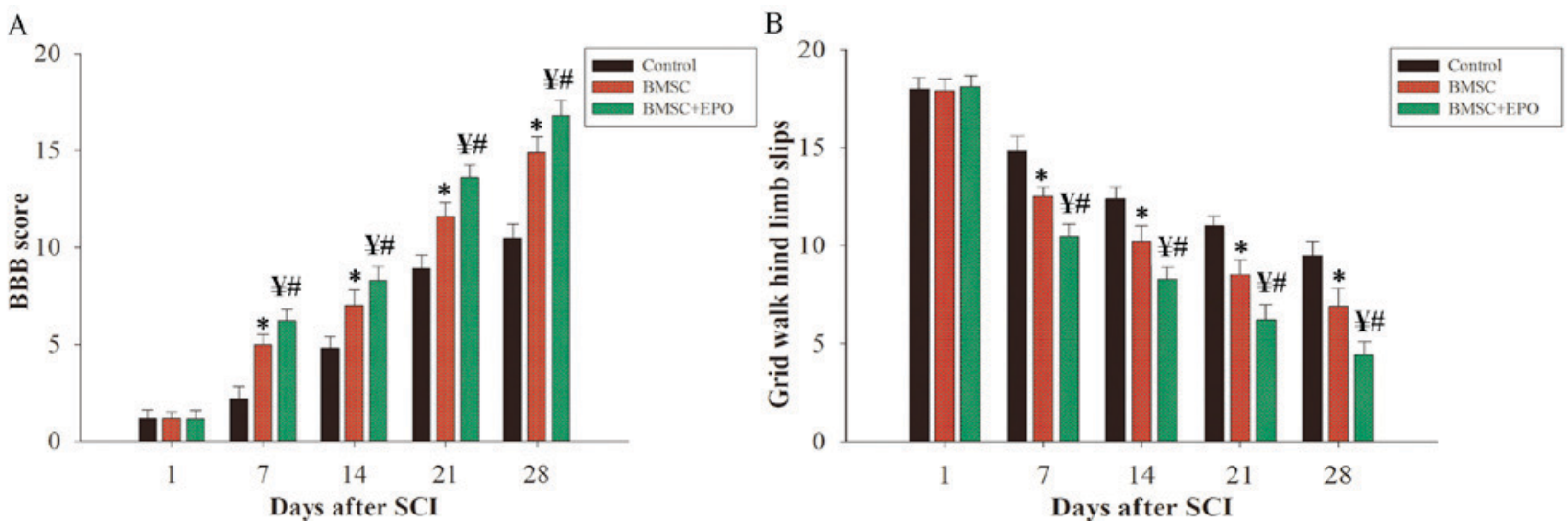

Figure 1. Locomotor function recovery after SCI in rat models treated with BMSCs or BMSCs + EPO. (A) BBB scale scores, which represent the restoration of hind limb function post-SCI. (B) Grid walk test of hind limbs post-SCI to evaluate motor sensitivity and capacity for precise control of the hind limbs. Results are presented as the mean \pm standard deviation. ${ }^{*} \mathrm{P}<0.05$ vs. control group; ${ }^{{ }^{7}} \mathrm{P}<0.05$ vs. BMSCs group; ${ }^{\sharp} \mathrm{P}<0.01$ vs. control group. SCI, spinal cord injury; BMSCs, bone marrow mesenchymal stem cells; EPO, erythropoietin; BBB, Basso, Beattie and Bresnahan locomotor scale.

A

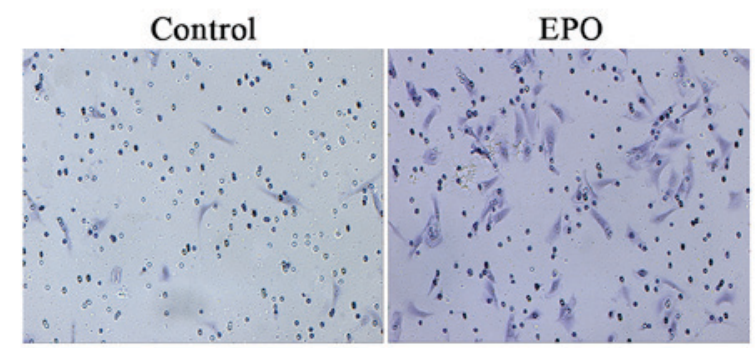

B

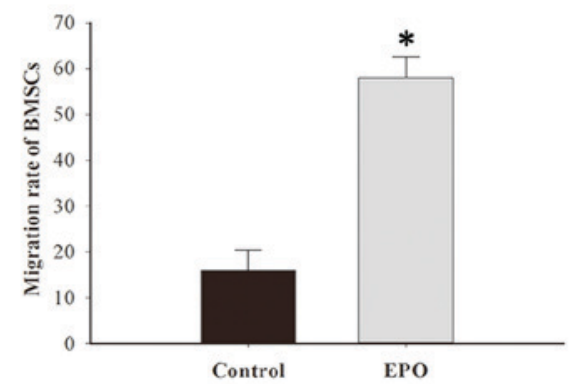

Figure 2. Effect of EPO on improving the migration capacity of BMSCs in vitro. (A) Images of the BMSCs under a light microscope, magnification $\mathrm{x} 400$. The BMSCs were dyed with crystal violet and the cells transplanted were violet. (B) Transwell migration assay results. Cells were measured three times, then the average was taken. Results are presented as the mean \pm standard deviation. ${ }^{*} \mathrm{P}<0.001$ vs. BMSCs alone group. EPO, erythropoietin; BMSCs, bone marrow mesenchymal stem cells.

Effect of EPO on the migration capacity of BMSC in vitro. The transwell migration assay was performed to access the effect of EPO on the in vitro migration capacity of BMSCs (Fig. 2A). The results identified that EPO significantly increased the migration capacity of BMSCs compared with the BMSCs alone group ( $\mathrm{P}<0.001$; Fig. 2B).

Effect of EPO on the apoptotic index of SCI sites. The TUNEL assay was used to identify apoptotic cells and apoptotic cells were stained brown (Fig. 3A-C). This was used to determine the apoptotic index of the groups following SCI (Fig. 3D). There was no significant difference in the apoptotic indexes of the three groups 1 day following SCI (Fig. 3D). However, 7 and 28 days following SCI, the apoptotic index of the BMSC group was significantly decreased compared with the control group $(\mathrm{P}<0.05)$ and the apoptotic index of the BMSCs + EPO group was significantly decreased compared with the control $(\mathrm{P}<0.01)$ and BMSC treatment groups $(\mathrm{P}<0.05)$ (Fig. 3D). These results demonstrate that EPO may increase the anti-apoptotic effect of BMSCs in SCIs.

EPO facilitates the recruitment of BMSCs to sites of SCI. An immunofluorescence assay was performed on SCI samples taken 4 weeks following SCI (Fig. 4). Fluorescence signals were almost undetectable in the control group (Fig. 4A), while GFP-labeled BMSCs were detected at the site of the SCI in the BMSC treatment (Fig. 4B) and BMSC + EPO groups (Fig. 4C). However, the signal was stronger in the BMSC + EPO group (Fig. 4C). These results indicate that EPO facilitates the migration of BMSCs to sites of SCI.

$E P O$ increases in vivo expression of VEGF and BDNF. The expression of VEGF and BDNF was determined through western blotting (Fig. 5A) and subsequent image analysis (Fig. 5B). The BMSC treatment group had significantly increased VEGF and BDNF expression levels compared with the control group $(\mathrm{P}<0.05)$, and expression levels in the BMSC + EPO group were significantly higher compared with those of the control $(\mathrm{P}<0.01)$ and BMSC treatment groups $(\mathrm{P}<0.05)$.

\section{Discussion}

The determining factor for the therapeutic efficacy of BMSC transplantation is not the quantity of engraftments, but the quantity of BMSCs that successfully migrate to the lesion site (21). In addition, a number of grafting routes increase the quantity of successful BMSCs and enhance the restoration of neurological function following SCI, such as transplanting directly into the lesion site, through the cerebrospinal fluid or through caudal vein (22).

At the site of SCI there is upregulation in the expression of numerous chemokines, cytokines, corresponding receptors and growth factors, including VEGF, platelet-derived growth factor, hepatocyte growth factor and tumor necrosis factor- $\alpha$ (23). These factors, together with signaling molecules 
A

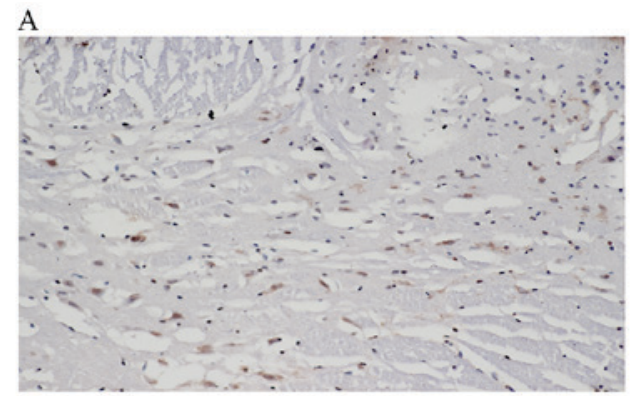

$\mathrm{C}$

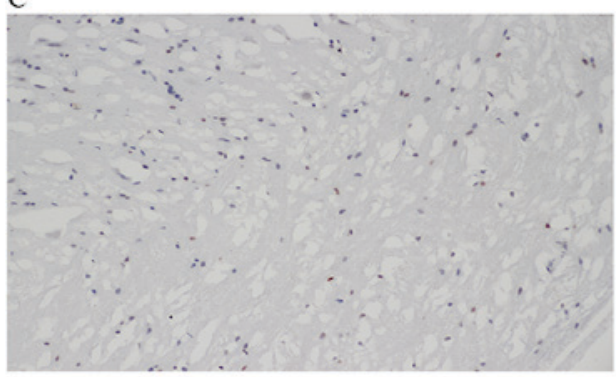

B
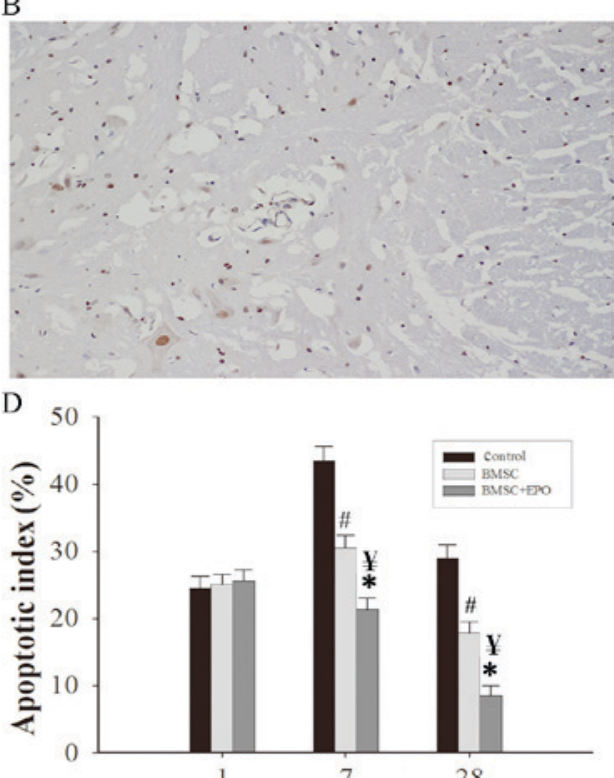

Days after SCI

Figure 3. Effect of EPO on the apoptotic index of SCI sites. Images showing apoptosis in the sites of SCI 7 days post-SCI at x400 magnification in (A) the control, (B) the BMSC treatment group and (C) the BMSCs + EPO group. Apoptotic cells were stained dark brown, normal nuclei were blue. (D) Apoptotic index (percentage of apoptotic cells) in each group following SCI. The number of apoptotic cells was decreased in the BMSCs + EPO group compared with the control and BMSC treatment groups. Results are presented as the mean \pm standard deviation. ${ }^{*} \mathrm{P}<0.01$ vs. control group, ${ }^{{ }^{*}} \mathrm{P}<0.05$ vs. BMSC group, ${ }^{~} \mathrm{P}<0.05$ vs. control group. SCI, spinal cord injury; BMSC, bone marrow mesenchymal stem cells; EPO, erythropoietin.
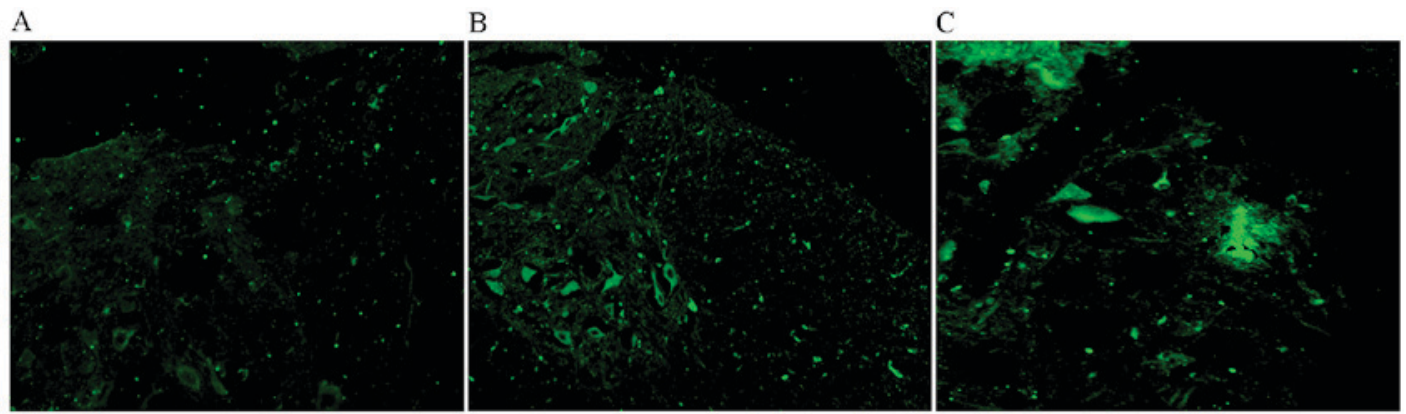

Figure 4. Effect of erythropoietin (EPO) on the localization of bone marrow mesenchymal stem cells (BMSCs) transplanted into spinal cord injury (SCI) sites 28 days following SCI in (A) the control group, (B) the BMSC treatment group and (C) the BMSC + EPO group. GFP-labeled BMSCs were observed at the site of SCI in the BMSC and BMSC + EPO groups.

A

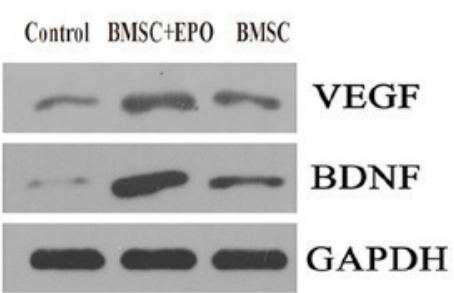

B

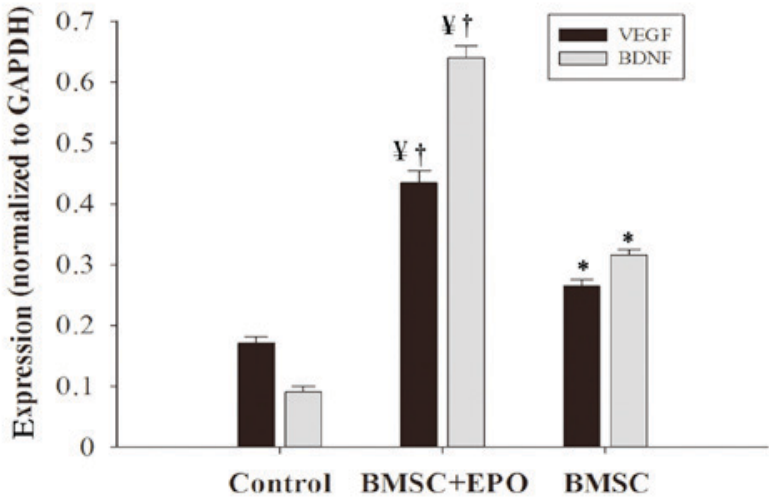

Figure 5. Expression of VEGF and BDNF 28 days following spinal cord injury. Protein expression levels were determined through (A) western blotting followed by (B) analysis. Expression levels were normalized to GAPDH. Results are presented as the mean \pm standard deviation. " $\mathrm{P}<0.05$ vs. control; ${ }^{\dagger} \mathrm{P}<0.05$ vs. Control, ${ }^{*} \mathrm{P}<0.05$ vs. BMSC group. VEGF, vascular endothelial growth factor; BDNF, brain derived neurotrophic factor; BMSC, bone marrow mesenchymal stem cell; EPO, erythropoietin. 
in the local microenvironment, form a signaling network that directs the migration of BMSCs in vivo (24). However, it is difficult to evaluate the validity of biomaterials and BMSC treatment for SCI, as there is a deficiency in controlled studies and methodology guidance.

The primary restrictions in the therapeutic application of BMSCs for SCI are poor cell viability following transplantation, a deficiency in neuronal differentiation, necrosis, glial scar formation, poor microenvironment and the short time period in which transplantation is successful (25-27). In order to overcome these restrictions, a number of strategies have been applied, including stimulating neuronal differentiation prior to grafting, neurotrophic gene transfection, co-administration of glial cells and histological engineering (28-30). In a previous study, genetically engineered human BMSCs injected above and below the SCI site in rats immediately following SCI significantly ameliorated subsequent locomotor function (31). In addition, transplantation of neurally differentiated BMSCs into the center of a contused spinal cord in rats resulted in restoration of locomotor function and significantly shortened primary latency of BMSCs (32). Co-transplantation of neurally differentiated and undifferentiated autologous BMSCs into the site of SCI in rats remarkably improved BBB locomotor scale scores compared with controls (33). Results of the present study indicate that EPO promotes the migration of BMSCs to SCI sites and significantly improves locomotor function following SCI.

Angiogenesis is essential to increase blood flow to injured areas, a crucial component of neural reconstruction following SCI. VEGF stimulates angiogenesis and increases vascular permeability, which is important in the healing of SCIs (34). A previous study in rats indicated that microvessel density is significantly increased by administration of VEGF, which improved locomotor function (35). BMSCs secrete VEGF and BDNF, which trigger downstream signaling by binding to the tropomyosin-receptor-kinases A and B (36). In addition, a previous study found that VEGF produced by BMSCs induced angiogenesis and osseous regeneration (37). Furthermore, Han et al (38) identified that administration of simvastatin promotes expression of BDNF and VEGF, which regulates the microenvironment of the injured spinal cord, resulting in improved viability, proliferation and neuronal differentiation of BMSCs recruited to the site of SCI. In the present study, co-administration BMSC + EPO upregulated the expression of BDNF and VEGF at the site of SCI and promoted recovery of neurological function following SCI.

Neuronal apoptosis is an essential part of the pathophysiological process following SCI. BMSCs serve a primary role in the inhibition of apoptosis by stimulating endogenous salvage signaling pathways and secreting anti-apoptotic factors (39). EPO has been determined to inhibit neuronal apoptosis in a rat model of compressional SCI (40). Furthermore, a previous study found that EPO significantly inhibited apoptosis of motor neurons in transient spinal cord ischemia (41). In the present study, BMSC transplantation significantly decreased apoptosis compared with the control group, while co-transplantation of BMSC + EPO significantly decreased apoptosis compared with the control and BMSC treatment groups. This indicates that EPO enhances the anti-apoptotic effect of BMSCs.
In conclusion, the present study suggests that EPO facilitates the recruitment of BMSCs to sites of SCI, increases the expression of BDNF and VEGF, enhances the anti-apoptotic effect of BMSCs and accelerates recovery of neurological function following SCI. These results indicate that the co-transplantation of $\mathrm{BMSC}+\mathrm{EPO}$ is a promising strategy for the treatment of traumatic SCIs.

\section{Acknowledgements}

The present study was supported by the Natural Science Foundation of Hubei Province, Hubei, China (grant no. 2013CFC035).

\section{References}

1. Hagen EM: Acute complications of spinal cord injuries. World $\mathrm{J}$ Orthop 6: 17-23, 2015.

2. Sekhon LH and Fehlings MG: Epidemiology, demographics, and pathophysiology of acute spinal cord injury. Spine (Phila Pa 1976) 26 (24 Suppl): S2-S12, 2001.

3. Morawietz $\mathrm{C}$ and Moffat F: The effects of locomotor training after incomplete spinal cord injury: A systematic review. Arch Phys Med Rehabil 94: 2297-2308, 2013.

4. Bustos ML, Huleihel L, Kapetanaki MG, Lino-Cardenas CL, Mroz L, Ellis BM, McVerry BJ, Richards TJ, Kaminski N, Cerdenes N, et al: Aging mesenchymal stem cells fail to protect because of impaired migration and antiinflammatory response. Am J Respir Crit Care Med 189: 787-798, 2014.

5. Ansboro S, Greiser U, Barry F and Murphy M: Strategies for improvement of therapeutic cells: Implications for tissue repair. Eur Cells Mater 23: 310-319, 2012.

6. Kean TJ, Duesler L, Young RG, Dadabayev A, Olenyik A, Penn M, Wagner J, Fink DJ, Caplan AI and Dennis JE: Development of a peptide-targeted, myocardial ischemia-homing, mesenchymal stem cell. J Drug Target 20: 23-32, 2012.

7. Martinez AM, Goulart CO, Ramalho Bdos S, Oliveira JT and Almeida FM: Neurotrauma and mesenchymal stem cells treatment: From experimental studies to clinical trials. World J Stem Cells 6: 179-194, 2014.

8. Patel DM, Shah J and Srivastava AS: Therapeutic potential of mesenchymal stem cells in regenerative medicine. Stem Cells Int 2013: 496218, 2013.

9. Robert CR, Sorkin M, Garg RK and Gurtner GC: Stem cell recruitment after injury: Lessons for regenerative medicine. Regen Med 7: 833-850, 2012.

10. Novikova LN, Brohlin M, Kingham PJ, Novikov LN and Wiberg M: Neuroprotective and growth-promoting effects of bone marrow stromal cells after cervical spinal cord injury in adult rats. Cytotherapy 13: 873-887, 2011.

11. Hoffmann J, Glassford AJ, Doyle TC, Robbins RC, Schrepfer S and Pelletier MP: Angiogenic effects despite limited cell survival of bone marrow-derived mesenchymal stem cells under ischemia. Thorac Cardiovasc Surg 58: 136-142, 2010.

12. Eliopoulos N, Zhao J, Forner K, Birman E, Young YK and Bouchentouf M: Erythropoietin gene-enhanced marrow mesenchymal stromal cells decrease cisplatin-induced kidney injury and improve survival of allogeneic mice. Mol Ther 19: 2072-2083, 2011.

13. Xiong M, Chen S, Yu H, Liu Z,Zeng Y and Li F: Neuroprotection of erythropoietin and methylprednisolone against spinal cord ischemia-reperfusion injury. J Huazhong Univ Sci Technolog Med Sci 31: 652-656, 2011.

14. Teixeira M, Rodrigues-Santos P, Garrido P, Costa E, Parada B, Sereno J, Alves R, Belo L, Teixeira F, Santos-Silva A and Reis F: Cardiac antiapoptotic and proproliferative effect of recombinant human erythropoietin in a moderate stage of chronic renal failure in the rat. J Pharm Bioallied Sci 4: 76-83, 2012.

15. Chen S, Li J, Peng H, Zhou J and Fang H: Administration of erythropoietin exerts protective effects against glucocorticoid-induced osteonecrosis of the femoral head in rats. Int $\mathrm{J}$ Mol Med 33: 840-848, 2014.

16. Liu NM, Tian J, Wang WW, Han GF, Cheng J, Huang J and Zhang JY: Effect of erythropoietin on mesenchymal stem cell differentiation and secretion in vitro in an acute kidney injury microenvironment. Genet Mol Res 12: 6477-6487, 2013. 
17. Nair AM, Tsai YT, Shah KM, Shen J, Weng H, Zhou J, Sun X, Saxena R, Borrelli J Jr and Tang L: The effect of erythropoietin on autologous stem cell-mediated bone regeneration. Biomaterials 34: 7364-7371, 2013

18. Onifer SM, Zhang O, Whitnel-Smith LK, Raza K, O'Dell CR, Lyttle TS, Rabchevsky AG, Kitzman PH and Burke DA: Horizontal ladder task-specific re-training in adult rats with contusive thoracic spinal cord injury. Restor Neurol Neurosci 29: 275-286, 2011.

19. Deng W, Bivalacqua TJ, Chattergoon NN, Jeter JR Jr and Kadowitz PJ: Engineering ex vivo-expanded marrow stromal cells to secrete calcitonin gene-related peptide using adenoviral vector. Stem Cells 22: 1279-1291, 2004.

20. Basso DM, Beattie MS and Bresnahan JC: A sensitive and reliable locomotor rating scale for open field testing in rats. J Neurotrauma 12: 1-21, 1995.

21. Nakano N, Nakai Y, Seo TB, Homma T, Yamada Y, Ohta M, Suzuki Y, Nakatani T, Fukushima M, Hayashibe M and Ide C: Effects of bone marrow stromal cell transplantation through CSF on the subacute and chronic spinal cord injury in rats. PLoS One 8: e73494, 2013.

22. Caplan AI and Correa D: PDGF in bone formation and regeneration: New insights into a novel mechanism involving MSCs. J Orthop Res 29: 1795-1803, 2011.

23. Urdzíková LM, Růžička J, LaBagnara M, Kárová K, Kubinová Š Jiráková K, Murali R, Syková E, Jhanwar-Uniyal M and Jendelová P: Human mesenchymal stem cells modulate inflammatory cytokines after spinal cord injury in rat. Int J Mol Sci 15: 11275-11293, 2014.

24. Xia P, Pan S, Cheng J, Yang M, Qi Z, Hou T and Yang X: Factors affecting directional migration of bone marrow mesenchymal stem cells to the injured spinal cord. Neural Regen Res 9: 1688-1695, 2014.

25. Nakajima H, Uchida K, Guerrero AR, Watanabe S, Sugita D, Takeura N, Yoshida A, Long G, Wright KT, Johnson WE and Baba H: Transplantation of mesenchymal stem cells promotes an alternative pathway of macrophage activation and functiona recovery after spinal cord injury. J Neurotrauma 29: 1614-1625, 2012.

26. Mothe AJ, Bozkurt G, Catapano J, Zabojova J, Wang X, Keating $\mathrm{A}$ and Tator $\mathrm{CH}$ : Intrathecal transplantation of stem cells by lumbar puncture for thoracic spinal cord injury in the rat. Spinal Cord 49: 967-973, 2011.

27. Boido M, Garbossa D, Fontanella M, Ducati A and Vercelli A Mesenchymal stem cell transplantation reduces glial cyst and improves functional outcome after spinal cord compression. World Neurosurg 81: 183-190, 2014

28. Abrams MB, Dominguez C, Pernold K, Reger R, Wiesenfeld-Hallin Z, Olson L and Prockop D: Multipotent mesenchymal stromal cells attenuate chronic inflammation and injury-induced sensitivity to mechanical stimuli in experimental spinal cord injury. Restor Neurol Neurosci 27: 307-321, 2009.
29. Osaka M,Honmou O,Murakami T,Nonaka T,Houkin K,Hamada H and Kocsis JD: Intravenous administration of mesenchymal stem cells derived from bone marrow after contusive spinal cord injury improves functional outcome. Brain Res 1343: 226-235, 2010.

30. Cho SR, Kim YR, Kang HS, Yim SH, Park CI, Min YH, Lee BH, Shin JC and Lim JB: Functional recovery after the transplantation of neurally differentiated mesenchymal stem cells derived from bone barrow in a rat model of spinal cord injury. Cell Transplant 18: 1359-1368, 2009.

31. Zhang YJ, Zhang W, Lin CG, Ding Y, Huang SF, Wu JL, Li Y, Dong $\mathrm{H}$ and Zeng YS: Neurotrophin-3 gene modified mesenchymal stem cells promote remyelination and functional recovery in the demyelinated spinal cord of rats. J Neurol Sci 313: 64-74, 2012.

32. Feng Y, Wang J, Ling S, Li Z, Li M, Li Q, Ma Z and Yu S Differentiation of mesenchymal stem cells into neuronal cells on fetal bovine acellular dermal matrix as a tissue engineered nerve scaffold. Neural Regen Res 9: 1968-1978, 2014.

33. Pedram MS, Dehghan MM, Soleimani M,Sharifi D, Marjanmehr SH and Nasiri Z: Transplantation of a combination of autologous neural differentiated and undifferentiated mesenchymal stem cells into injured spinal cord of rats. Spinal Cord 48: 457-463, 2010.

34. Canavese M and Spaccapelo R: Protective or pathogenic effects of vascular endothelial growth factor (VEGF) as potential biomarker in cerebral malaria. Pathog Glob Health 108: 67-75, 2014.

35. Patel CB, Cohen DM, Ahobila-Vajjula P, Sundberg LM, Chacko T and Narayana PA: Effect of VEGF treatment on the blood-spinal cord barrier permeability in experimental spinal cord injury: Dynamic contrast-enhanced magnetic resonance imaging. J Neurotrauma 26: 1005-1016, 2009.

36. Wyse RD, Dunbar GL and Rossignol J: Use of genetically modified mesenchymal stem cells to treat neurodegenerative diseases. Int J Mol Sci 15: 1719-1745, 2014.

37. Chen J, Zhang C, Jiang H, Li Y, Zhang L, Robin A, Katakowski M, Lu M and Chopp M: Atorvastatin induction of VEGF and BDNF promotes brain plasticity after stroke in mice. $\mathrm{J}$ Cereb Blood Flow Metab 25: 281-290, 2005.

38. Han X, Yang N, Cui Y, Xu Y, Dang G and Song C: Simvastatin mobilizes bone marrow stromal cells migrating to injured areas and promotes functional recovery after spinal cord injury in the rat. Neurosci Lett 521: 136-141, 2012.

39. Isele NB, Lee HS, Landshamer S, Straube A, Padovan CS, Plesnila N and Culmsee C: Bone marrow stromal cells mediate protection through stimulation of PI3-K/Akt and MAPK signaling in neurons. Neurochem Int 50: 243-250, 2007.

40. Arishima Y, Setoguchi T, Yamaura I, Yone K and Komiya S Preventive effect of erythropoietin on spinal cord cell apoptosis following acute traumatic injury in rats. Spine (Phila Pa 1976) 31: 2432-2438, 2006

41. Celik M, Gökmen N, Erbayraktar S, Akhisaroglu M, Konakc S, Ulukus C, Genc S, Genc K, Sagiroglu E, Cerami A and Brines M: Erythropoietin prevents motor neuron apoptosis and neurologic disability in experimental spinal cord ischemic injury. Proc Natl Acad Sci USA 99: 2258-2263, 2002. 